Décadrages Décadrages

cınéma, à travers champs Cinéma, à travers champs

3 | 2004

Hitchcock côté cour

\title{
Les reprises du dispositif narratif de Rear Window chez Brian De Palma
}

Alain Boillat

\section{(2) OpenEdition}

Journals

Édition électronique

URL : http://journals.openedition.org/decadrages/555

DOI : $10.4000 /$ decadrages. 555

ISSN : 2297-5977

Éditeur

Association Décadrages

Édition imprimée

Date de publication : 10 avril 2004

Pagination : 45-58

ISBN : 978-2-9700582-0-5

ISSN : 2235-7823

Référence électronique

Alain Boillat, "Les reprises du dispositif narratif de Rear Window chez Brian De Palma », Décadrages

[En ligne], 3 | 2004, mis en ligne le 29 avril 2013, consulté le 01 mai 2019. URL : http://

journals.openedition.org/decadrages/555; DOI : 10.4000/decadrages.555

(B) Décadrages 


\section{Les reprises du dispositif narratif de Rear}

\author{
Window chez Brian De Palma
}

\author{
par Alain Boillat
}

J'apprenais à communiquer selon la grammaire bitchcockienne, à utiliser ses intrigues, ses personnages. [...] Afin de comprendre ses méthodes de travail, et ayant de mon côté des idées très influencées par ses constructions originales et ingénieuses, $j$ 'ai commencé à faire des films ressemblant à des méditations sur Psychose, Vertigo, etc. [...] Je savais ce que je faisais lorsque je travaillais sur les thèmes ["ideas»] de Hitchcock. Je me fichais qu'on m'accuse de plagiat ["imitator»] ou de choquer les gens parce que ça se fait dans l'art depuis la nuit des temps. En réalité, j'étais un disciple de Hitchcock, comme si j'avais été en cours avec lui. Je le comprenais mieux, je voyais ce qu'il voyait. Je me fichais de ce que les critiques pensaient, parce que la plupart des critiques ont dix ans de retard sur ce qui se fait.

Brian De Palma

\section{De Palma et le pastiche hitchcockien}

Dans cet extrait d'un entretien récent ${ }^{\mathbf{1}}$, le réalisateur Brian De Palma commente le rapport qu'il entretenait avec l'œuvre d'Alfred Hitchcock dans les années 70 et 80 , période durant laquelle il s'est essentiellement illustré dans un genre spécifique, le thriller. Pour parler de l’héritage hitchcockien, il reprend des arguments qu'il avance depuis Sours de sang (Sisters, 1972), contraint d'adopter une attitude défensive ou une pseudo-indifférence face à la critique américaine qui l'accusait de piller Hitchcock de manière éhontée. Sa justification s'origine dans l'affirmation d'une connaissance intime des films du "Maître» qui lui permettrait de s'identifier à ce dernier et l'autoriserait plus que tout autre à juger de ses propres innovations ${ }^{2}$. Souvent, De Palma tente d'expliquer sa démarche à l'aide de comparaisons qui ont trait soit à
1 De Palma les années 60, documentaire de Luc Lagier et Amaury Voslion, Arte, 2002-2003, disponible sur le DVD Brian De Palma années 60, GCTHV, 2003. Nous reproduisons ici le texte qui figure en sous-titre dans cette édition.

2 "lls ne pensent pas comme lui. Et je peux comprendre à quel point ce qu'il faisait était génial. [...] Moi, je comprends pourquoi Hitchcock a choisi tel plan plutôt qu'un autre, je peux mesurer l'étendue de son génie " (in Brian De Palma, Entretiens avec Samuel Blumenfeld et Laurent Vachaud, Editions Calmann-Lévy, Paris, 2001). 
3 Dans Obsession (1976), dont l'histoire coécrite avec Paul Schrader se présente ouvertement comme une variation sur Vertigo (Sueurs froides, A. Hitchcock, 1958), Sandra (Geneviève Bujold), de laquelle s'éprend son père qui ne l'a pas reconnue et qui croit retrouver en cette jeune femme son épouse décédée, travaille à la restauration d'un retable de Daddi dans une église florentine. Cet espace constitue justement le lieu de la première rencontre avec la mère dont le mausolée est aujourd'hui orné d'une réplique miniature de la façade de cette église. Sandra y explique à l'Américain qu'il existe sous la surface de l'œuvre en restauration une peinture plus ancienne que les experts ont décidé de sacrifier au profit du retable. Cette substitution du nouveau à l'ancien fait écho au récit répétitif du film (la beauté retrouvée de la Madone restaurée est aussi celle de la mère disparue), mais peut aussi être comprise comme une mise en abyme du rapport du cinéaste à son modèle (en ce sens, le récit de Vertigo contient en lui-même l'idée de pastiche). Par le biais du palimpseste se révèle le fantasme de remplacer l'œuvre première. Significativement, "l'original" n'est pas pour Sandra la fresque plus ancienne, mais l'œuvre de Daddi. Le lapsus de cette réplique renvoie au tabou dont est entachée la filiation, l'inceste suggéré par le film (le père épouse sa fille) faisant office d'équivalent métaphorique du plagiat. Ce désir de surpasser l'original est clairement affirmé par De Palma à propos d'Howard Hawks, dont il réalise en 1983 un remake du film Scarface (1931): "Au bout du compte, je suis convaincu que mon Scarface dépassera le film de Hawks, pourtant celui-ci est plutôt bon, mais à l'arrivée vous verrez, quand on parlera de Scarface, c'est à mon film qu'on fera référence" (Brian De Palma, Entretiens avec Samuel Blumenfeld et Laurent Vachaud, op. cit., p. 112). Si, dans le cas particulier et institutionnalisé d'un remake, l'emprunt à un héritage passé est légitimé, De Palma ne s'est probablement pas risqué à être aussi explicite à propos de Hitchcock. II n'en pense pas moins lorsqu'il affirme à propos de la filiation Vertigo-Obsession: "L'idée est la même c'est sûr, mais pour être franc, je trouve mon histoire meilleure" (id., p. 53).

4 Par exemple, De Palma recourt déjà à cette terminologie ("vocabulaire", "grammaire", etc.) dans un entretien donné au Los Angeles Times après la sortie d'Obsession (entretien cité par Robert Kapsis, Hitchcock, the Making of a Reputation, University of Chicago, Chicago, d'autres domaines artistiques, notamment la peinture $\mathbf{3}$ où la question de l'imitation des anciens ne serait pas dévaluée, soit au langage verbal, la "grammaire» élaborée par Hitchcock constituant selon lui une sorte de fonds commun que chaque cinéaste peut se réapproprier ${ }^{4}$. Il est significatif que le réalisateur présente son travail comme une "méditation" sur les films de Hitchcock. En effet, ceux-ci constituent pour lui un point de départ, une matrice susceptible de générer des développements narratifs et esthétiques spécifiques. Les films de Brian De Palma ne sont pas seulement des adaptations au goût du jour de thèmes hitchcockiens (notamment en ce qui concerne la représentation plus explicite de la violence et du sexe), mais sont construits sur des éléments qui, une fois empruntés, subissent une importante reformulation et contribuent à interpréter le film-source 5 . D'ailleurs, la référence cinématographique n'est jamais unique, chaque film procédant d'un croisement d'emprunts divers. Si, par exemple, Body Double (1985) part de Rear Window, c'est pour rejoindre ensuite la filature et l'expérience de la perte vécue par Scottie dans Vertigo, la claustrophobie remplaçant chez De Palma le vertige. Même Obsession (1976) qui revisite explicitement l'ensemble de la trame narrative de Vertigo intègre également certains passages d'autres films (notamment Rebecca). Ce mode cumulatif de la citation découle de l'affirmation auteuriste propre à Hitchcock où le film singulier s'offre, selon Katie Trumpener ${ }^{6}$, comme la métonymie de l'ensemble de «l'œuvre du Maître».

On le voit, la pratique du pastiche est essentielle pour aborder le fonctionnement des films de Brian De Palma. Le terme ne doit pas être confondu avec la parodie, dont De Palma n'use selon moi que dans un seul film de la période, Le fantôme du paradis (1974) qui, en détournant certains stéréotypes du film d'horreur et des thèmes issus de Faust et du Fantôme de l'opéra (et non de films de Hitchcock!), rappelle le ton plus léger et irrévérencieux de ses comédies des années 60. Pour définir sommairement le pastiche, on peut dire, à la suite de Genette ${ }^{7}$, qu'il se caractérise par une imitation qui s'opère certes sur un régime ludique, mais qui n'endosse pas de fonction satirique ou comique. Chez Brian De Palma, les éléments empruntés sont toujours transposés dans un contexte sérieux $\mathbf{8}$ et subissent une véritable intégration. Le caractère ludique du pastiche participe chez lui d'une visée plus large: il s'agit d'exhiber une maîtrise technique qui est poussée à son paroxysme dans des scènes de bravoure où, au risque de briser la cohérence narrative, certains procédés (plan-séquence, écran partagé, ralenti, montage rapide, etc.) sont particulièrement soulignés et récurrents comparativement aux productions de ses contemporains tels que Coppola, Scorsese ou Lucas. Cette 
grandiloquence conduit à un fort degré d'auto-réflexivité, le film s'affichant au regard d'un spectateur abandonné à cette virtuosité comme un objet construit et illusoire, à l'opposé de l'idéal de la narration dite classique qui viserait à la "transparence» des procédés discursifs. Au sein du cinéma "classique", Alfred Hitchcock fait de toute manière figure d'exception dans la mesure où sa "marque d'auteur» tend à atténuer le déni des processus d'élaboration même du film. Cette trace se manifeste de manière évidente (quoique brève et ludique) dans les apparitions du cinéaste. Comme pour Lubitsch, les maisons de production n'ont d'ailleurs pas hésité à utiliser cette touche personnelle comme argument promotionnel. John Belton dit à ce propos que «la visibilité d'Hitchcock en tant que narrateur est devenue un des éléments de la relation contractuelle avec son public»9. C'est pourquoi on trouve déjà chez Hitchcock de nombreuses scènes de bravoure (les plans-séquences de La corde, la scène du manège dans l'Inconnu $d u$ Nord-Express, de l'avion dans La mort aux trousses ou de la douche dans Psycho) qui sont manifestement marquées par des signes de maîtrise destinés à provoquer une réaction cinéphilique. Certains choix formels résultent d'une motivation qui est d'ordre "artistique» selon la typologie de David Bordwell10, et non liée à des enjeux narratifs ou à la psychologie des personnages. Brian De Palma ne fait qu'accentuer cette composante déjà présente dans l'œuvre pastichée. Rear Window est d'ailleurs le film qui, le plus ostensiblement, parle autant de ce qu'il raconte que du cinéma même. Les effets du pastiche s'inscrivent donc dans une certaine continuité avec les éléments pastichés tout en augmentant, ne serait-ce que par l'opération même de transposition, leur degré d'auto-réflexivité.

En affirmant cette continuité, je vais à l'encontre d'une conception partagée par nombre de critiques selon lesquels le cinéma de Brian De Palma pourrait être qualifié de "post-moderne", un terme qui me paraît être bien souvent une étiquette fourre-tout. Ce que l'on pointe avec cette notion a néanmoins effectivement trait à notre problématique dans la mesure où elle est censée rendre compte d'un cinéma qui instaure un nouveau rapport à l'héritage de ses pères, notamment sous la forme du pastiche. Cependant, bien que De Palma appartienne à la "génération Lucas-Spielberg» à laquelle Laurent Jullier11 attache une certaine idée du post-modernisme, je ne crois pas que son cinéma ressortisse de ce paradigme, car, à l'exception de son film d'école Woton's Wake (1962) et de quelques cas ponctuels dans ses longs métrages - notamment ceux qui peuvent être considérés comme ses "grosses productions» $\mathbf{1 2}$-, aucun de ses films ne participe d'un "cinéma de l'allusion» où la citation n'est que pur clin d'œil. Sans aller jusqu'à
1992, p. 198). Dans l'un des entretiens réalisés par Blumenfeld et Vachaud (op. cit., p. 91), le cinéaste exprime la même idée: "l'utilisation de la grammaire hitchcockienne est innée chez moi et je n'y réfléchis pas du tout". Cette comparaison linguistique rejoint la distinction saussurienne entre langue et parole.

5 C'est ce qu'illustre Nicole Brenez dans une démarche figurale à l'aide des citations depalmiennes de la scène de la douche de Psycho dans De la figure en général et du corps en particulier, De Boeck, Paris/Bruxelles, 1998, p. 322-335.

6 Katie Trumpener, "Fragments of the Mirror", Hitchcock's Released Films, Raubichek et Srebnik (éd.), Wayne State University Press, Detroit, 1991, p. 176.

7 Gérard Genette, Palimpsestes. La littérature au second degré, Seuil, coll. "Points Essais", Paris, 1982, p. 39-47.

8 II est intéressant de noter que pour Obsession et Pulsions, films autour desquels s'est cristallisée la position hostile de la critique américaine qui s'est mise à voir les films de De Palma comme des plagiats de l'œurre hitchcockienne, c'est justement l'absence d'humour et de dimension parodique qui est relevée (cf. citations données par Robert Kapsis, op. cit., p. 197 et p. 202-203). Selon ces critères, la parodie est évaluée positivement de par la forte distance qu'elle introduit, alors que le pastiche est considéré comme une imitation stérile.

9 John Belton, "Spectacle and Narrative", Alfred Hitchcock's Rear Window, J. Belton (éd.), Cambridge University Press, Cambridge/ New York, 2000, p. 10.

10 Cf. The Classical Hollywood Cinema, David Bordwell, Janet Staiger, Kristin Thompson (éd.), Routledge, New York, 1985, p. 21. Bordwell exemplifie la "motivation artistique" avec $L a$ corde d'Hitchcock, mais évoque aussi la parodie, avec laquelle le pastiche à la De Palma présente à cet égard une certaine parenté.

11 Laurent Jullier, L'écran post-moderne. Un cinéma de l'allusion et du feu d'artifice, L'Harmattan, Paris, 1997.

12 Comme le landau qui dévale les escaliers lors d'une fusillade dans Les Incorruptibles (1987), allusion à la scène des escaliers d'Odessa du Cuirassé Potemkine d'Eisenstein, ou le voleur suspendu à un fil dans la chambre forte de Langley dans Mission: impossible (1996), qui rappelle Topkapi de Jules Dassin. 
13 Fredric Jameson, "Post-modernism and Consumer Society", Movies and Mass Culture, John Belton (éd.), Rutgers University Press, New Brunswick, 1996, p. 190-194.

Notons que Jameson, l'un des plus célèbres théoriciens du post-modernisme, considère Brian De Palma dans Signatures of the visible (Routledge, New York/Londres, 1992, p. 54-55) comme l'équivalent américain de Jean-Jacques Beineix dont il analyse le film Diva. Cette comparaison me paraît abusive, le rapport nostalgique à la référence ne me semblant pas être du même ordre chez les deux cinéastes. La pensée de Jameson n'est toutefois pas réductible à cette seule étude de cas, et certains points relatifs au phénomène de la réception post-moderne qu'il aborde ailleurs s'appliquent également au travail de Brian De Palma.

Pour une taxinomie des différentes positions théoriques relatives au post-modernisme en fonction de leurs postulats esthétiques et politiques, cf. Fredric Jameson, Postmodernism, or The Cultural Logic of Late Capitalism, Verso / Duke University Press, Londres/New York, 1999, notamment p. 55-66.

14 Herrmann étant le compositeur attitré d'Alfred Hitchcock, de L'homme qui en savait trop (1956) à Marnie (1964), sa musique est associée par le spectateur, fût-ce inconsciemment, à la période la plus célèbre de la carrière d'Alfred Hitchcock. C'est d'ailleurs essentiellement aux années 50 que Brian De Palma se réfère.

15 Lorsque j'entends "Hitchcock", je ne le considère quant à moi pas comme une personne particulière ("le créateur", "l'auteur"), les "dispositifs narratifs " dont il est question ici devant également beaucoup aux différents scénaristes avec lesquels le réalisateur collabora, mais comme une entité qui permet de désigner un corpus de films célèbres auxquels on rattache généralement un certain nombre de caractéristiques communes. Mon optique ne vise donc pas tant à défendre une position auteuriste qu'à partir de celle-ci, dans la mesure où elle constitue un aspect déterminant de la production (Hitchcock se constituant en tant que star) et de la réception, de même qu'une composante de l'intertexte volontairement généré par d'autres films (en l'occurrence ceux de Brian De Palma). Voir la conclusion "L'auteur est un réseau " de Jean-Pierre Esquenazi, Hitchcock et l'aventure de Vertigo. L'invention à Hollywood, Editions CNRS, Paris, 2001. s'inscrire dans un processus de déconstruction critique, la référence cinématographique n'y est pas réduite à une complicité ludique avec le spectateur (qu'elle n'exclut cependant pas), mais travaille en profondeur le film et conditionne les phénomènes de compréhension narrative à l'œuvre chez le spectateur, au-delà d'une simple reconnaissance de l'élément pastiché. Quant à la nostalgie, selon Fredric Jameson $\mathbf{1 3}$ caractéristique d'un cinéma post-moderne (dont American Graffiti de Lucas serait le parangon) qui exprimerait l'impossibilité de toute innovation par son enfermement délibéré dans le passé, on ne peut pas dire qu'elle s'observe chez De Palma au niveau de l'univers représenté, puisque ce dernier se compose généralement de lieux et d'outillages contemporains (appartements design, équipement bigh-tech, etc.). Certains éléments purement visuels relèvent certes ponctuellement d'une telle démarche, telle la saturation des couleurs (évoquant le Technicolor des années cinquante) ou l'utilisation d'une profondeur de champ et d'un éclairage rappelant les transparences hitchcockiennes (notamment lors de trajets en voiture), mais cette composante nostalgique me semble subordonnée à un projet plus vaste de renouvellement narratif qui exploite le pastiche de manière productive. C'est pourquoi il me semble intéressant de s'interroger sur les principes du «recyclage» depalmien, notamment sur la nature des éléments empruntés aux films de Hitchcock au-delà d'une certaine "ambiance» qui est en fait une question de genre, et que le recours au musicien Bernard Herrmann ${ }^{\mathbf{1 4}}$ pour Sours de sang et Obsession ne fait que souligner.

Il y a d'une part des références ponctuelles à certains procédés de prise de vue (par exemple le mouvement circulaire autour des amants dans Vertigo que l'on retrouve dans Body Double), d'autre part la reprise de fragments de récit, ou plutôt de ce que je proposerais d'appeler des "dispositifs narratifs". C'est sur ce dernier point que nous quittons la superficialité de la simple allusion. En effet, lorsque De Palma évoque, dans la citation liminaire, son intérêt pour les «constructions originales et ingénieuses» d'Alfred Hitchoock ${ }^{\mathbf{1 5}}$, il met en évidence un trait qui leur est commun : la manipulation du spectateur. En effet, comme dans le flash-back initial mensonger du Grand alibi (Stagefright, 1950), nombre de films de Brian De Palma comportent une amorce ou un prologue qui désoriente le spectateur en se dévoilant rétrospectivement comme un leurre (le "film dans le film" dans Sours de sang, Blow Out et Body Double, la succession de deux mises en scène dans Mission: impossible, les actions filmées en plan-séquence au début de Snake Eyes ensuite décortiquées à l'aide des bandes enregistrées par les caméras de surveillance, la fusée du premier plan de Mission to Mars). Un tel choix pour des débuts de films, 
"moments contractuels» par excellence ${ }^{\mathbf{1 6}}$ où se noue le rapport au spectateur, est tout à fait révélateur d'une intention de jouer avec ce dernier (qui, après le générique, sait à quoi s'attendre), à l'instar de Hitchcock, soucieux de maintenir le suspense, élément central de l'horizon d'attente construit par ses films. Le pastiche concourt à renforcer cette complicité ludique avec le public en définissant un horizon d'attente précis via les références à des films connus, puis à s'en écarter en modifiant certains paramètres. En parlant de «dispositif» narratif, j'entends une modalité spécifique de gestion de la transmission de l'information narrative, l'assignation d'une "place» donnée au spectateur au sein de l'univers filmique qui passe notamment par la construction d'un système qui relie regards et actants du récit. La plupart des «grandes scènes» réalisées par De Palma, en général axées sur l'imminence d'une menace, reposent sur une telle configuration 17. Celle de la place de l'église montrée à deux reprises dans Femme fatale (2001) est emblématique à cet égard, car les divers éléments du drame s'inscrivent ostensiblement dans un réseau médiatisé par des regards, la réception des rayons solaires faisant office de chaîne causale: le photographe du mariage, dans l'attente d'une lumière adéquate, lève les yeux en direction du soleil dont les rayons se réfléchissent ensuite sur la valise métallique de l'héroïne avant d'atteindre le pendentif transparent du camionneur qui, aveuglé, provoque l'accident impliquant la complice et ses poursuivants. Cette scène est en outre soumise au regard surplombant du reporter assis sur son balcon qui la reconstitue par ses clichés pris depuis un lieu unique.

En général, la tension s'instaure entre une action mettant un personnage en péril et ceux qui en sont les témoins passifs, incapables d'intervenir (à l'image du spectateur de cinéma qui n'a pas prise sur le déroulement du récit). Ce type de situations et le voyeurisme qu'elles supposent (ou, sans la dimension perverse, l'observation) s'avèrent récurrents chez De Palma18 et présentent, bien sûr, une parenté fondamentale avec Rear Window. Il serait toutefois exagéré de ramener chaque occurrence d'un tel dispositif au film de Hitchcock, car on en trouverait dans presque chaque film de Brian De Palma, ainsi que dans quantité d'autres films qui ne se donnent pas comme des pastiches de Rear Window. Le pastiche de Rear Window ne peut être considéré comme tel que s'il met en scène un personnage observant depuis son propre appartement des voisins à leur insu (du moins selon ce qu'il présume), sans aucune intention d'épier une action particulière. Afin de me concentrer sur des éléments qui me semblent nécessaires à la reconnaissance par le spectateur du dispositif pastiché, j'écarte les cas de filature où un personnage en espionne un autre, comme lorsque le «fantôme du Paradis» épie Swan et Phoenix
16 Sur cette notion, voir André Gardies, Le récit filmique, Hachette, Paris, 1993, p. 44-45.
17 Par exemple la scène du bal dans Carrie (1976), où les divers protagonistes sont "disposés" autour du seau rempli de sang prêt à basculer, ou dans de la salle de billard de Carlito's Way (L'impasse, 1993), l'observation du personnage connaissant une dilatation temporelle qui retarde l'explosion de la violence.

18 Comme l'affirme De Palma sur un plan très général: "Le cinéma, c'est ça: voir, voir, voir. Le thème du voyeurisme est un thème éminemment cinématographique. Epier à travers une fenêtre, c'est une parfaite métaphore du cinéma" (entretien de 1994, in Les Inrockuptibles, n० 54, cité par Dominique Legrand, Brian De Palma, le rebelle manipulateur, Editions du Cerf, Paris, 1995, p. 202). 
19 Notons que dans Le fantôme du Paradis, le voyeur est lui-même observé par Swan qui actionne une caméra située à l'extérieur, de sorte que sa position d'extériorité est mise en péril. Ce déplacement de l'espace du spectacle (l'action centrale se déroulera ensuite sur la toiture lorsque Winslow Leach tentera de se suicider), révélé par un split screen, provoque un retournement de situation qui est du même ordre que la manipulation de Body Double. On rencontre également cette situation de "l'épieur épié" à un certain stade du récit de Rear Window, lorsque Lars Thorwald fixe la caméra, renvoyant au statut de voyeur non seulement Jefferies, mais aussi le spectateur du film.

20 Je pense aux caméras de surveillance lors de l'assaut de la villa de Tony Montana dans Scarface, au point de vue offert par le dirigeable miniature en forme d'énorme œil dans Snake Eyes, aux moniteurs-montres de Mission: impossible, etc.

21 Dans la seconde partie, la situation tend à s'inverser en plaçant Rubin devant la caméra, d'abord celle des organisateurs de la pièce d'avant-garde anti-raciste Be Black Baby dans laquelle il joue le rôle d'un policier, puis celle de la télévision venue interviewer des voisins de l'immeuble qui a fait l'objet d'un attentat à la bombe (en fait perpétré par John).

22 Comme l'acteur-personnage n'a pas encore été montré (contrairement à l'introduction de La Dame du lac de Robert Montgomery, 1947), l'identification au personnage ne peut supplanter l'identification première à la caméra, liée au pur plaisir de voir (sur ces notions, cf. Christian Metz, Le Signifiant imaginaire, Christian Bourgois, Paris, 1993 [1977], p. 66-72).

23 La longue scène de l'attaque d'une guérilla urbaine est filmée en noir et blanc avec un surcadrage figurant les bords arrondis d'un écran TV. La scène s'arrête d'ailleurs lorsque John Rubin sort une arme et tire sur son téléviseur, alors que le film même se termine sur le mode du gag lorsque le même personnage, filmé par une équipe TV, salue sa mère (ce qui donne son titre au film).

24 Cette auto-désignation de "Godard américain " apparaît fréquemment dans les déclarations faites par le cinéaste à l'époque, et fait office d'appellation dans la périodisation proposée par Robert Kapsis (op. cit., p. 190) pour la période 1965-1975. Notons qu'on ne fait pas tant allusion au Godard qui est exactement contemporain des films de De Palma, mais plutôt à

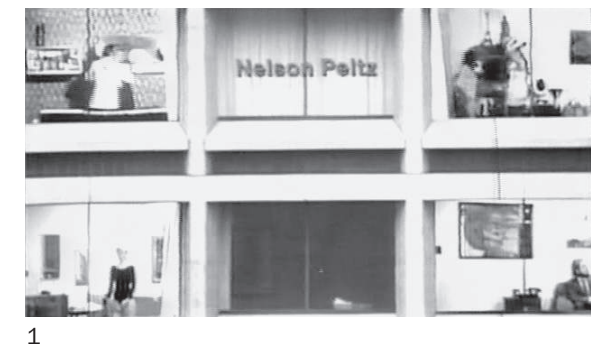

enlacés depuis les toits à travers une verrière 19, ou, à la fin de Pulsions, quand Peter observe de loin le cabinet du Dr. Elliot. Je laisse également de côté les nombreuses scènes 20 qui, chez De Palma, impliquent une position de voyeur par rapport à des écrans (même si, métaphoriquement, le champ libéré par les stores qui se lèvent au début de Fenêtre sur cour redouble la surface écranique) pour axer les quelques remarques qui vont suivre sur les trois films suivants: Hi, Mom! (1969), Saurs de sang (1972) et Body Double (1985).

\section{La comédie du voyeur}

Dans Hi, Mom!, De Palma poursuit l'histoire d'un des personnages de Greetings (1968), le voyeur invétéré John Rubin (joué dans les deux films par Robert De Niro). Dans la dernière séquence du premier film, on l'avait quitté en soldat américain lors de la guerre du Viêt-Nam, alors qu'il contraignait une jeune Vietnamienne à se déshabiller devant la caméra de la TV qui l'interviewait. Après l'avoir observée dans le viseur de son fusil, il lui demandait de mimer la présence d'une fenêtre par laquelle elle était censée s'offrir au regard du filmeur. Totalement recréée, la situation voyeuriste renvoie indubitablement à la pathologie du personnage. Cette mise en scène, totalement incongrue parmi les sifflements de balles, trouve sa place dans le milieu urbain de $\mathrm{Hi}$, Mom!

Le personnage de Rubin n'existe d'ailleurs dans la première partie de ce film que comme sujet d'un regard $\mathbf{2 1}$ : la séquence de pré-générique durant laquelle il visite un logement miteux est strictement tournée en caméra subjective, de sorte que l'action même de regarder importe plus que la mise en place du personnage 22. Le visage de De Niro n'apparaît significativement qu'au moment où il est question de la vue qu'offre l'une des fenêtres. Il est révélé au spectateur lorsque le rideau qui cachait la vitre est arraché, c'est-à-dire au moment où l'on s'attendrait à voir un plan de l'immeuble d'en face. Cette substitution cantonne le personnage dans son rôle de voyeur, d'un être qui ne peut exister que comme contre-champ de ce qu'il observe. C’est pourquoi le générique 
ne démarre qu'à cet instant de constitution du "Je», faisant alterner, sous les mentions écrites, des plans de l'immeuble d'en face et d'autres montrant John Rubin s'essayant à filmer depuis sa chambre.

$H i, M o m$ ! est donc placé sous la référence à Rear Window dès son générique où le champ est subdivisé en six cases constituées par les fenêtres d'une façade, dont quatre «s'allument» progressivement, affichant le caractère non-réaliste de cette vue. Les fenêtres y sont avant tout de petits écrans (fig. 1), en accord avec le monde obnubilé par la télévision décrit par ce film qui pratique sans cesse la mise en abyme ${ }^{\mathbf{2 3}}$. Toutefois, contrairement au film de Hitchcock, Hi, Mom! fait fi de tout sérieux: déluré et discontinu, il est caractéristique de cette époque où Brian De Palma se considérait comme une sorte de "Godard américain» $\mathbf{2 4}$. Les micro-actions que Rubin capte dans les appartements des voisins, toutes plus ou moins liées à la généralisation des techniques de prise de vues amateur (l'un essaie une caméra $8 \mathrm{~mm}$, l'autre un nouvel appareil photo, etc.), donnent une image d'une société totalement exhibitionniste et voyeuriste, mais ne génèrent aucun développement narratif. Décousus, les moments épiés sont d'ailleurs filmés en accéléré, ce qui introduit une défétichisation de l'objet du regard, dès lors condamné à la banalité et au ridicule de la répétitivité mécanique. Par ailleurs, l'intérêt du voyeur n'est orienté que par l'aspect sexuel de ce qui peut se présenter à lui, puisqu'il a obtenu de travailler pour un producteur de films érotiques (un lien qui sera poursuivi dans Body Double) auquel il a promis d'offrir "de l'art, l'art d'épier les gens». Nous sommes donc à des lieues de l'intrigue policière de Rear Window. Non satisfait de ce qu'il surprend, Rubin passe d'ailleurs de voyeur à acteur en tentant de filmer ses ébats avec une voisine à l'insu de celle-ci, ce qui occasionne une situation comique où il doit gérer l'empressement de sa compagne en fonction du moment de déclenchement automatique de sa caméra. Ce timing exprime la fusion recherchée par le protagoniste hédoniste entre observation et érotisme, une thématique qui, dans Rear Window, n'est que suggérée par les plans sur la danseuse (Mlle Torso), et globalement refoulée par le traitement de la question du mariage 25 . L'auto-mise en scène de Rubin destinée à la caméra et la possibilité de différer le vu et le voir grâce à l'enregistrement donnent lieu à une représentation beaucoup plus active du voyeur qui entraîne la manipulation effective de la voisine. L'impuissance de Jefferies qui, ayant un membre dans le gypse, se contente d'observer, n'est pas à l'œuvre ici, contrairement à Body Double dans lequel la scène de voyeurisme fait écho à la séquence d'ouverture où le protagoniste surprend sa femme avec un amant, après une séquence pré-générique qui l’a montré incapable de jouer son rôle la période de son travail antérieur à La Chinoise (1967), notamment aux films communément associés à l'esprit de la Nouvelle Vague comme A bout de souffle ou Pierrot le fou qui, justement, recourent massivement à la citation.

25 Dans Rear Window, de nombreux parallèles s'instaurent entre la ligne d'actions principale qui a trait à ce qu'observe Jefferies, et la ligne secondaire (dont la subordination est à discuter, puisque la portée de tous les événements du film peut être reversée sur elle) qui touche à l'intimité des relations de couple entre Jefferies et Lisa, cette dernière ne cessant d'aborder leur mariage qu'il refuse: les petites scènes qui se déroulent dans les appartements d'en face rejouent selon différentes modalités ou à divers stades (la solitude, les jeunes mariés, le vieux couple, etc.) ce que pourrait être cette relation conjugale que Jefferies craint (peutêtre jusqu'au point de choisir le meurtre que Thorwald commet symboliquement à sa place). L'intrication des pistes narratives repose donc sur la bifurcation qui s'opère entre le désir érotique et le plaisir scopique, le second ne pouvant être interprété dans les termes du premier, comme l'indique la phrase de la nurse qui, vérifiant le thermomètre, raille Jefferies en disant que "les pin-up d'en face n'ont pas fait monter [sa] température". Spectateur de ces divers possibles, Jefferies s'y réfugie, s'y projette pour oublier l'espace de son propre appartement et les ardeurs de sa compagne. Cette attitude est particulièrement évidente dans une scène où Lisa l'embrasse et tente d'attirer son attention alors qu'il ne cesse de regarder dehors, la bifurcation s'effectuant explicitement dans le dialogue à partir du terme ambigu "problème" (Jefferies: "Don't you ever have any problems?") qui renvoie, pour Lisa, à l'indifférence de son amant, pour Jefferies au meurtre présumé qui l'obsède. Peu après, Jefferies reste à la fenêtre alors que Lisa s'étend sur le lit: ces deux sous-espaces renvoient aux deux types de désir, irréconciliables jusqu'au moment où Lisa entrera dans la logique du voyeur en s'intéressant au meurtre et en se rendant dans l'appartement d'en face, c'est-à-dire en devenant elle-même l'objet du regard. Un autre quiproquo verbal apparaît dans la même scène: contemplant Mlle Torso étendue sur son lit en tenue légère (une nuisette noire et transparente comme la robe de Lisa à ce moment-là, ce qui souligne le parallélisme), Jefferies énonce que "ça doit être un sacré boulot..." (on pense à celle qui doit plaire aux hommes, comme cela 
a été dit auparavant), puis poursuit après une pause, sans lien (apparent) avec Mlle Torso, en disant "...comment découperais-tu un cadavre?". Refoulant le corps comme objet du désir, Jefferies ne peut que le concevoir de façon sadique et morbide. Cet exemple illustre les implications psychologiques importantes du croisement entre la romance et l'intrigue policière.
26 L'héroïne exerce donc une profession parente de celle de Jefferies dans Rear Window, mais qui déplace la question de la vue (il est photo-reporter) dans le domaine de l'écrit, ce qui la prédispose moins explicitement à la position de voyeur. Par ailleurs, si Jefferies et Grace sont tous deux des personnages d'action, ils n'agissent pas sur le même plan: il est un aventurier, elle est une militante, comme en témoigne la réaction des policiers qui refusent d'intervenir parce que Grace a dénoncé leur inefficacité dans la presse. Remarquons que Sisters recourt, comme Rear Window, à une visualisation qui naturalise l'exposition apprenant au spectateur le métier du personnage: De Palma nous montre des articles affichés dans la pièce. L'identification du personnage via sa nomination fonctionne également comme le nom "Jefferies" écrit sur le plâtre: les articles sont signés "Grace Collier" alors que simultanément la protagoniste s'annonce hors-champ au téléphone.

27 Sur ces points (le rapport aux autorités policières, la place de la femme, la question raciale, etc.), voir Armond White, "Eternal Vigilance in Rear Window", Alfred Hitchcock's Rear Window, op. cit., p. 126-131. de vampire lors du tournage d'un film. Dans Hi, Mom!, le voyeur se trouve dans une position de domination absolue (d'ailleurs, il n'est pas qu'acteur, mais aussi réalisateur), du moins jusqu'au moment où la technique passe au poste de commande: lorsque la caméra fixée sur un trépied pivote accidentellement vers le bas, révélant un autre appartement dans lequel un Blanc se grime avec de la peinture noire, c'est le film tout entier qui bascule dans un autre sujet, celui de la troupe «Be Black Baby» qui met ses spectateurs dans la peau des Noirs pour leur faire expérimenter l'oppression raciale.

\section{Sisters: les écrans siamois}

$\mathrm{Si} H \mathrm{H}, \mathrm{Mom}$ ! recourt au dispositif de Rear Window dans un genre qui ne travaille nullement la référence à l'œuvre de Hitchcock (il s'agit d'une comédie, et non d'une parodie), Sisters inaugure la pratique du pastiche dans le domaine du thriller inspiré du «Maître», d'une part en reprenant dans la scène nodale du meurtre la situation d'un témoin qui a "fenêtre sur» le crime, d'autre part en accentuant dans le sens des films d'horreur (slasher, gore, etc.) et de la drug culture seventies l'intrigue psychanalysante de Spellbound ou de Psycho. La composition musicale très typée de Bernard Herrmann renforce l'allusion à l'atmosphère des films les plus célèbres de Hitchcock.

La séquence qui nous intéresse est celle où une journaliste 26 , Grace Collier (Jennifer Salt), qui n'a peut-être de "Kelly» que le "collier», surprend en regardant par sa fenêtre l'agonie d'un jeune homme noir qui a été poignardé par Danielle (Margot Kidder), sa conquête de la veille qui, prise d'une crise de schizophrénie, a ressuscité en elle sa sœur jumelle Dominique morte des années auparavant. Comme Jefferies, Grace passera tout le film à tenter de prouver qu'il y a bien eu un assassinat. Audelà des différences qui ont trait au contexte socio-historique ${ }^{27}$, j'aimerais avancer quelques remarques sur le fonctionnement de ce dispositif narratif chez De Palma comparativement à Rear Window.

Il faut tout d'abord noter que, contrairement à Rear Window où, excepté quelques brefs passages, le point de vue est strictement limité à celui de Jefferies $\mathbf{2 8}$, le spectateur de Sisters n'occupe pas la position du témoin dès les premières images du film: ce n'est qu'après presque une demi-heure, au moment où la victime en sang arrive à ramper jusqu'à la fenêtre, que le personnage de Grace est introduit. Cette mise en place tardive du dispositif restrictif accorde au spectateur une certaine omniscience, et exclut tout doute quant à la vérité du témoignage - et ainsi toute culpabilité du voyeur -, alors que Jefferies peut être longtemps suspecté d'avoir affabulé ce qu'il raconte (comme l'épouse jouée par 
Joan Fontaine dans Soupçons). Jennifer Salt ne joue pas véritablement le rôle d'un voyeur dans le sens où elle n'observe pas sciemment ses voisins jusqu'à ce que quelque chose se produise, mais son attention est happée par l'atrocité du crime. Dans Rear Windowe, le meurtre a lieu horschamp, lorsque Jefferies est endormi, et le spectateur ne fait qu'entendre, comme lui, un cri de femme, alors que dans Sisters le «spectacle» du crime est exhibé dans son caractère le plus sanglant. Le thème du voyeurisme est par contre exposé dans ce film dès le pré-générique, puisque Danielle rencontre son amant lors d'un jeu télévisé significativement nommé «Peeping Tom» dans lequel elle se fait passer pour aveugle et se déshabille devant lui, les spectateurs présents dans le studio devant deviner quelle sera la réaction de l'observateur. Ce bref «film dans le film» expose la nature illusoire de la position privilégiée qui consiste à voir sans être vu, annonçant le piège tendu au protagoniste masculin de Body Double.

$\mathrm{La}$ «focalisation zéro» $\mathbf{2 9}$ qui domine en ce début de film atteint justement son acmé lors de cette scène qui relie deux fenêtres d'immeubles adjacents à travers un procédé plutôt rare au cinéma mais fréquent chez De Palma, le split screen ("écran partagé»). En effet, le cinéaste a déjà usé systématiquement de cette technique dans son documentaire tourné avec deux caméras, Dionysus in '69 (1969), dans le but de présenter simultanément une performance théâtrale et l'espace du public progressivement intégré à la «scène». Mêler indissociablement le spectacle et son effet sur une instance d'observation (qui est diégétique, les appareils de prise de vue demeurant toujours invisibles) semble être au cœur de l'esthétique du réalisateur. Dans Sisters, au moment même où l'on passe de la victime au témoin (d'abord présent, flou, en arrière-plan), l'écran se divise en deux ( fig. 2 et 3), déployant l'espace dans une vue panoptique qui englobe les deux faces généralement disjointes du champ/contrechamp. Cet ancrage sur un regard extérieur est souligné par un zoom avant dans la partie droite (depuis l'appartement de Danielle), puis par un zoom arrière dans la partie gauche (dans le sens du témoin), de sorte
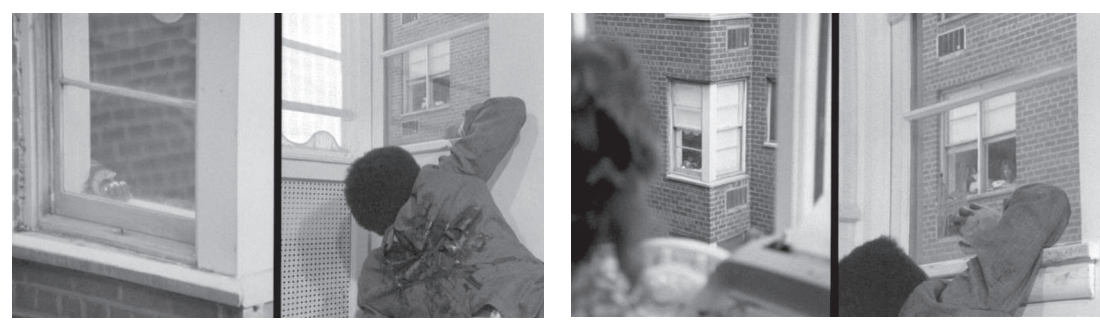

2
28 Même si Rear Window n'est pas tourné avec le procédé de la caméra subjective qui identifierait strictement le point de vue du spectateur à celui de Jefferies (qui dès lors peinerait à se constituer en tant que personnage), le spectateur n'a accès qu'à un nombre fort restreint d'informations inconnues de Jefferies, du moins parmi celles qui ont une véritable pertinence narrative. Une exception notable concerne cependant un plan unique, séparé par deux fondus, dans lequel un panoramique décrit un aller-retour entre Jefferies endormi dans sa chaise et l'immeuble d'en face vu de loin, où I'on voit Monsieur Thorwald quitter son appartement accompagné d'une (sa?) femme. Le mouvement de caméra permet de certifier que cette action a échappé au voyeur, incitant le spectateur du film à s'interroger sur le bien-fondé de ses spéculations (même si l'intertexte hitchcockien tend à accréditer l'hypothèse du meurtre). Une phrase de la nouvelle homonyme de William Irish correspond à ce passage de l'adaptation filmique: "Elle avait pu partir à mon insu, aux premières heures d'hier matin. Pendant quelques heures, j'avais dormi " (Fenêtre sur cour, Le Livre de Poche, Paris, 1975, p. 23). Ainsi les assoupissements de Jefferies ne permettent-ils pas seulement de jouer sur le temps en introduisant des ellipses, mais aussi sur la gestion de la transmission narrative.

29 Dans la typologie genettienne, la "focalisation zéro" correspond à un cas de figure où le savoir n'est pas médiatisé par un protagoniste de l'histoire, le narrateur en disant plus que n'en savent les personnages (Figures III, Seuil, Paris, 1972, p. 206). 
30 La série A comprend 19 plans, dont une série de brefs inserts, alors que la série B n'est composée que de 6 plans. On oppose donc la panique du témoin à la désorientation de la coupable psychiquement instable. que ce regard est à la fois appelé par la victime mourante et confirmé par Grace. Une sorte de pacte se crée, qui expliquera l'opiniâtreté de la journaliste. Le split screen permet d'évacuer tout hors-champ, d'être à la fois dans l'intimité du voyeur et immergé dans ce qu'il voit, fantasme ubiquitaire qui obéit lui-même à une logique voyeuriste. La simultanéité des actions permet d'une part de créer un fort suspense (Grace arrivera-t-elle à temps pour confondre la criminelle et son psychiatre qui sont en train d'effacer toute trace de l'agression?), notamment via l'ambiguité induite par la monophonie (la stéréophonie aurait permis de latéraliser sur la gauche ou la droite la provenance supposée de certains sons), d'autre part de travailler sur le rythme, non seulement au sein de chaque série, mais aussi "horizontalement» entre les deux séries A (Grace à gauche) et $\mathrm{B}$ (le lieu du crime, à droite), car les changements de plans ne s'effectuent pas aux mêmes moments en A et B $\mathbf{3 0}$. Il est important que la répartition gauche/droite ne varie pas, car le témoin se situe là où se constitue pour le spectateur «l'origine» du regard si l'on adopte la lecture (occidentale) de gauche à droite. Alors que Grace est encore à proximité de sa fenêtre, le hors-champ laissé sur sa droite appelle l'autre écran, qui ne respecte toutefois pas l'échelle de plan correspondant à ce qu'elle est censée voir. Ensuite, au cours de son trajet jusqu'à l'appartement d'en face, un montage parallèle se met en place sans relation à un sujet percevant, si ce n'est à l'intérieur même de la série A qui comprend de nombreux inserts subjectifs. On le voit, le travail virtuose qu'effectue De Palma sur le split screen modifie totalement les données de base du film de Hitchcock, tout en reproduisant certains de ses éléments narratifs (par la suite, Grace envoie dans l'appartement d'en face un détective privé qu'elle suit avec des jumelles comme Jefferies le fait pour Lisa, celui-ci recourant par ailleurs aussi à un détective). En fragmentant l'écran, c'est-à-dire en réduisant sa surface et en multipliant les vues, De Palma replace certains enjeux de Rear Window dans l'ère de la transmission cathodique (le petit écran sur lequel on zappe, indissociablement lié à l'idée de surveillance). Le partage de l'écran n'est donc pas qu'une expression métaphorique de l'un des thèmes du film (la schizophrénie, à laquelle le spectateur est en quelque sorte poussé, comme le lecteur de cet article face à la prolifération des notes dans la colonne marginale), mais également un moyen de complexifier le dispositif hitchcockien alors arraché à son ferment classique (l'éthique du point de vue dont découle l'interrogation sur la culpabilité du voyeur et l'occultation du meurtre) pour être projeté dans l'éclatement flamboyant d'une société où les technologies visuelles sont omniprésentes. Le morcellement $\mathrm{du}$ point de vue unique de Rear Window dessine, pour reprendre une 


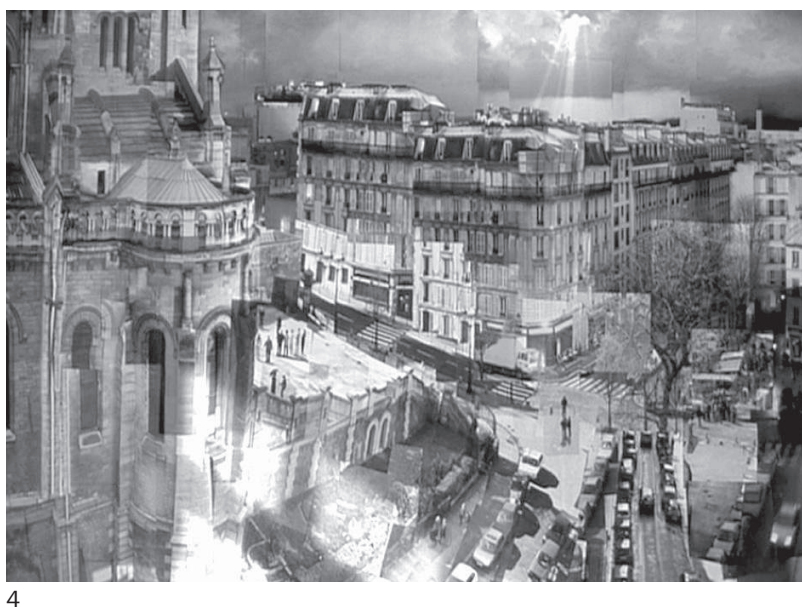

formule du cinéaste, une «tapisserie visuelle» $\mathbf{3 1}$ qui trouve son expression la plus emblématique dans la mosaïque des photographies que la caméra révèle progressivement à la faveur d'un travelling arrière dans l'ultime plan de Femme fatale (fig. 4). Toutefois, cet éclatement "cubiste" de l'image ne se présente pas comme une synthèse des divers possibles narratifs du film (même s'il renvoie, en tant que reconstruction de déroulements temporels, à l'aspect non-linéaire de la narration du film), mais conserve un centre (le reflet sur la mallette) et un foyer perceptif unique, celui du photo-reporter (Antonio Banderas) situé sur son balcon (qui, justement, apparaissait auparavant en split screen). Pour sa clôture, le film renoue donc avec l'unicité de Rear Window. Il n'est en ce sens pas indifférent que la "cour» de Femme fatale soit occupée par une cérémonie de mariage, puisqu'il s'agit du thème sans cesse rejoué dans le film de Hitchcock.

\section{Body Double: le dispositif comme machination}

Le film qui reprend une décennie plus tard le dispositif de Rear Window - et de façon plus littérale cette fois en mettant en jeu un véritable voyeur, mâle en quête d'une femme-objet qui s'exhibe -, Body Double, poursuit également le lien suggéré par Sisters avec la télévision (qui, rappelons-le, ouvrait le film): Jake Scully (Craig Wasson), après avoir vu périr atrocement la voisine qu'il avait contemplée à l'aide d'une longuevue alors qu'elle s'adonnait, devant les vastes vitres de son appartement, à des strip-teases nocturnes, reconnaît dans une émission TV érotique le modèle de celle qu'il avait épiée : la "voisine» était en fait une actrice, Holly Body, qui se livrait à un spectacle qui lui était spécifiquement
31 Expression utilisée par Brian De Palma pour décrire l'effet du split screen dans Pulsions (Brian De Palma, Entretiens avec Samuel Blumenfeld et Laurent Vachaud, op. cit., p. 96). 
32 De Palma tente de concilier le romantisme d'Hitchcock, auquel renvoie la musique quasi mystique et doucereuse des chœurs liée aux moments de contemplation à distance du corps de Gloria, avec une représentation plus crue de la sexualité (ce sont les masturbations de Gloria qui attirent Jake). Revenant sur son film, le cinéaste voit là l'une des "choses qui ne fonctionnent pas" (id., p. 123).

33 Célèbre, ce bâtiment (la Chemosphère de John Lautner, située à Los Angeles sur une des pentes qui borde Mulholland Dr.) attire l'attention du spectateur sur sa propre architecture et sa position surplombante, à l'instar du décor de la cour de Rear Window (le plus coûteux jamais construit à l'époque) qui constituait même un argument promotionnel pour le film. Ce quartier résidentiel huppé contraste avec le Greenwich Village de Fenêtre sur cour, comme s'il montrait l'envers de la vie des stars hollywoodiennes. Notons que l'organisation spatiale de la situation de voyeurisme réapparaît plus loin dans Body Double, d'abord dans un centre commercial à ciel ouvert (une place centrale entourée de vitrines de magasin), puis dans un motel situé au bord de la mer où, cette fois, Jake s'est vraiment rapproché de Gloria puisqu'il se trouve sur le balcon situé juste audessus du sien, et non en face. destiné, une doublure de celle dont il s'était épris, Gloria32. Jake en aura la confirmation en empruntant la cassette d'un des films pornographiques de Holly Body, nouveau fétiche dont il pourra répéter inlassablement certains passages lui rappelant la femme perdue. L'original n'est donc pas de l'autre côté de la cour, mais dans le petit écran: la fenêtreécran de la villa était déjà une duplication.

Comme dans le pré-générique de Sisters, le voyeur perd le pouvoir que lui confère l'anonymat en n'étant qu'un pion d'un dispositif qui lui échappe. Si le regard de Jake peut sembler acquérir une certaine maîtrise lorsqu'il découvre un étrange Indien, caricature du Méchant, espionnant lui aussi l'appartement de la Belle, c'est en fait tout le contraire: Jake voit ce que l'époux criminel, déguisé en Indien, a bien voulu qu'il voie afin qu'il accrédite en toute innocence, sur les traces du Scottie de Vertigo, une version erronée des faits. Ce mari, véritable metteur en scène, Jake l'a déjà rencontré, puisque c'est lui qui lui a proposé de séjourner dans cette splendide villa dominant de sa baie vitrée l'ensemble du quartier, "point de vue» idéal33. Attiré par le luxe de Hollywood, l'acteur minable en oublie le versant illusoire et destructeur. Dans le rôle de «l'entremetteur», le mari attise le désir de Jake en lui proposant de "se rapprocher un peu", et fait pivoter une lentille de la lunette permettant d'agrandir l'image (fig. 5 à 8), assouvissant cette pulsion scopique qui était aussi celle de Jefferies lorsqu'il fixait un téléobjectif sur son appareil photo. Mais à la différence du photo-reporter de Hitchcock, Jake n'agit pas seul, il est poussé à devenir un voyeur, puis à être témoin d'un meurtre qu'il est incapable d'empêcher. De Palma introduit un tiers dans la relation du voyeur à son objet, une instance qui est à la fois un deus ex machina et l'origine d'un regard dans le monde du film qui permet de renverser le dispositif: lorsque Jake et Holly Body discutent dans l'appartement, ils sont observés depuis l'autre immeuble par le meurtrier. Ainsi Jake-le-voyeur est-il lui-même montré dans une image cerclée de noir qui rappelle les plans où Jefferies surveille avec des jumelles Lisa entrée en infraction dans l'appartement des Thorwald. L'immunité du voyeur s'est perdue en passant de Jeff à Jake.
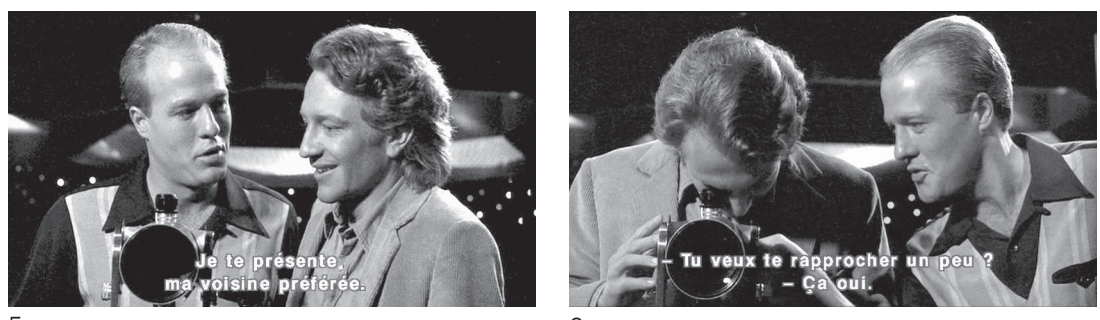

6 
L'assassinat à la perceuse électrique de Body Double, proche par son outrance de la tronçonneuse du film précédent (Scarface), visualise le hors-champ définitif de Rear Window (le meurtre de Madame Thorwald), comble une lacune en y déplaçant la scène de la douche de Psycho à laquelle la fragmentation des corps par le cadrage et le rythme effréné du montage font allusion. Cette scène violente, traumatique comme les films du passé pour un cinéphile obsessionnel, se présente sous la forme d'un collage de citations hitchcockiennes ${ }^{34}$. La scène de la douche suggérée ici réapparaît dans l'épilogue du film, plus fantasmé qu'effectif, où Jake reprend son rôle de vampire. Avant qu'il ne puisse faire mine de planter ses crocs dans la chair d'une jeune femme nue sous la douche, elle est remplacée par une doublure, escamotée comme Mme Thorwald. Si, dans Body Double, la femme de la villa d'en face semblait se donner entièrement au voyeur (contrairement à sa propre épouse qui se donnait à un autre), l'appropriation de l'objet du regard et du désir s'avère en fait impossible, se dérobant toujours au sujet dans cet intervalle de temps entre deux prises, imperceptible pour les spectateurs-voyeurs du film, mais bien réel. Le voyeur est confronté à l'inaccessibilité de son objet, d'autant plus imparable qu'il s'agit d'une illusion cinématographique. C'est pourquoi seule la mise en abyme, le «film dans le film", permet de combler l'absence de contact physique avec la réalité qui caractérise le voyeurisme (et le fétichisme qui en découle lorsque le voyeur ramasse dans une poubelle la petite culotte de Gloria qui devient après le décès de celle-ci explicitement le symbole du manque): Jake possède véritablement celle qu'il a désirée à distance sur le tournage d'un film pornographique, en se prêtant lui-même à une mise en scène. Ce n'est que dans ce contexte totalement fantasmatique que Holly Body - le corps sacré de Hollywood - peut se substituer à Gloria sous la forme d'un double qui conserve les attributs du fétiche. En déplaçant l'intrigue dans le milieu hollywoodien, De Palma prend l'auto-réflexivité de Rear Window à la lettre: tout est cinéma. C'est ce que ses films ne cessent de rappeler, notamment par le biais de la citation filmique.
34 Pour une analyse de ce tissu de citations, voir Jean-Philippe Trias, "Doublure \#1: le rêve du modèle", in Cinergon, n 16, 2003.

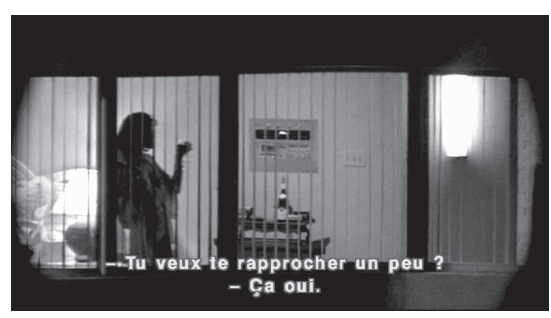

7

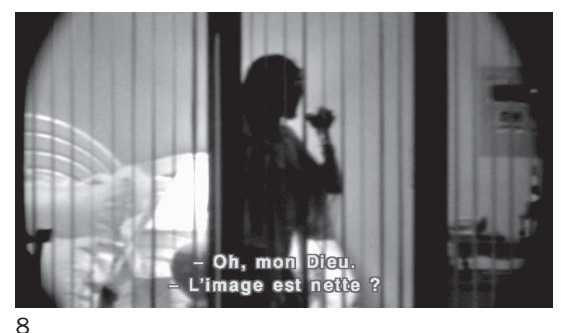

8 


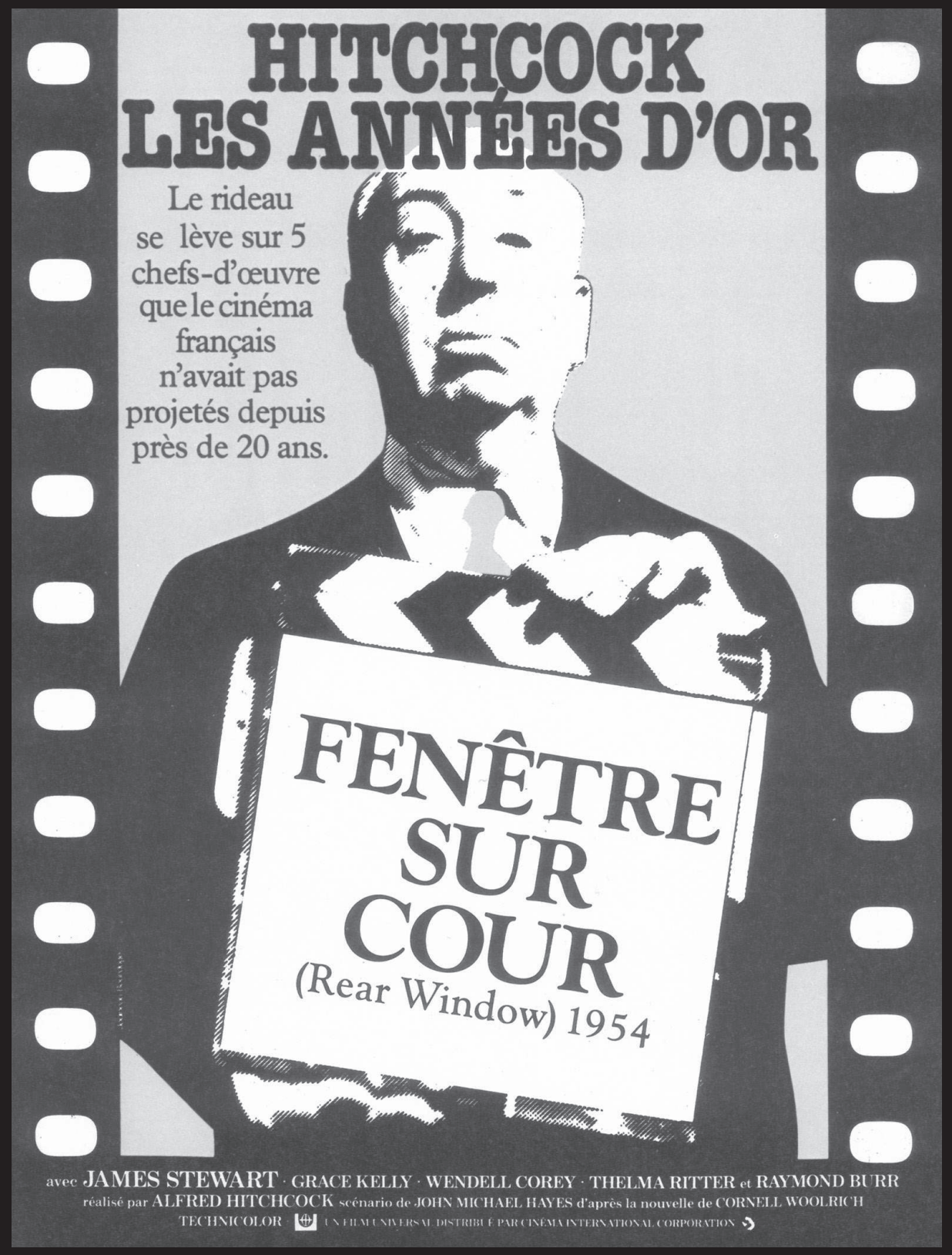

1984: RESSORTIE FRANÇAISE EN COPIES NEUVES APRÈS UNE ABSENCE DE 20 ANS. 